\title{
One Step Process for Infiltration of Magnetic Nanoparticles into CNT Arrays for Enhanced Field Emission
}

The complexities and functionalities of carbon nanotube (CNT) architectures can be enhanced even further by the addition of nanoparticles of different kinds. Here, a simple and easily scalable energy efficient infiltration technique is demonstrated to incorporate iron oxide $\left(\mathrm{Fe}_{3} \mathrm{O}_{4}\right)$ particles into aligned forests of CNTs grown by chemical vapor deposition. Scanning electron microscopy and in situ confocal microscopy confirm the presence of $\mathrm{Fe}_{3} \mathrm{O}_{4}$ and also explain the mechanism of infiltration and entrapment in the nanotube films. The obtained hybrid films are demonstrated to be excellent field emitters. The infiltration of nanoparticle results in an order of magnitude improvement in the turn-on field, which can be attributed to several advantageous factors such as reduced screening effects, improved conductivity, and local electric field enhancement in the proximity of the particles. The present method is generic and thus can be applied to other magnetic particles and porous host materials aiming at innovative sensor, electrical and environmental applications. (optical, electrical, magnetic, chemical, 12 and mechanical) even further, modifica- 13 tions by the means of encapsulation ${ }^{[10-13]} 14$ and surface anchoring of nanoparti- 15 cles of metals and metal oxides are 16 reported. ${ }^{[14-19]}$ Many efforts have been 17 devoted to decorating CNTs with diverse 18 materials by either covalent or van der 19 Waals attachment as well as by polymer 20 wrapping. ${ }^{[20]}$ Among the various nano- 21 composites, magnetic CNT composites 22 made of iron/iron oxide nanoparticles 23 were suggested to be of great importance 24 because of their potential uses in mag- 25 netic data storage, xerography, biosen- 26 sors, microwave absorbing materials and 27 in magnetic force microscopy. ${ }^{[21-24]}$ In 28 order to synthesize such composites, sev- 29 eral methods have been explored, such 30 as electron beam evaporation, chemical 31

\section{Introduction}

Carbon nanotubes (CNTs) due to their fascinating mechanical, electrical, thermal, and optical properties ${ }^{[1-4]}$ have emerged as promising candidates for a number of different nanoscale electronic devices. ${ }^{[5-9]}$ In order to innovative their properties

Dr. S. Sridhar, Dr. C. S. Tiwary, Dr. S. Ozden, Dr. K. Kalaga,

Dr. R. Vajtai, Prof. P. M. Ajayan

Department of Materials Science and NanoEngineering

Rice University

Houston, TX 77005, USA

E-mail: Robert.Vajtai@rice.edu; ajayan@rice.edu

Dr. C. S. Tiwary

Materials Science and Engineering

Indian Institute of Technology

Gandhinagar, India

Dr. B. Sirota, Prof. W. Choi

Department of Material Science and Engineering

University of North Texas

Denton, TX 76203, USA

Prof. K. Kordas

Microelectronics Research Unit

University of Oulu

P.O. Box 4500, FI-90014 Oulu, Finland

E-mail: krisztian.kordas@oulu.fi

The ORCID identification number(s) for the author(s) of this article can be found under https://doi.org/10.1002/admi.201701631.

DOI: 10.1002/admi.201701631 vapor deposition, hydrothermal growth, sol-gel synthesis, and 32 conventional impregnation. ${ }^{[14-25]}$ The decoration using most 33 of these processes are multistep and may compromise the 34 intrinsic characteristics of the CNTs.

In this paper, we report an easy, energy efficient, and scalable 36 method to prepare well-aligned CNTs decorated with magnetic 37 particles by using a unique magnetic infiltration technique as 38 shown in the schematic drawing in Figure 1. Besides its sim- 39 plicity, another major advantage of our proposed technique is 40 that the physical structure of the CNTs and the 3D forest align- 41 ment remains unaltered. We use in situ confocal microscopy 42 technique to understand the mechanism of such infiltration. 43 Further, we demonstrate stable field-emission of electrons with 44 a tremendous reduction in the turn-on field of devices. ${ }^{[25]} \quad 45$

\section{Q6}

\section{Results and Discussion}

Figure 2a-c shows SEM images of the CNTs with iron oxide 50 $\left(\mathrm{Fe}_{3} \mathrm{O}_{4}\right)$ particles infiltrated using our new method. The vertical 51 alignment and macroscopic structure of the 3D CNT remains 52 unchanged during the process. The SEM images from top and 53 side surfaces reveal the presence of iron oxide particles all around 54 the external surface of the film. From the low magnification 55 SEM image, we can clearly see the way in which the iron oxide 56 nanoparticles are distributed uniformly across the top of the 57 CNT array (marked by the arrow in Figure 2a). As explained in 58 the Experimental Section, the iron oxide particles got infiltrated 59 

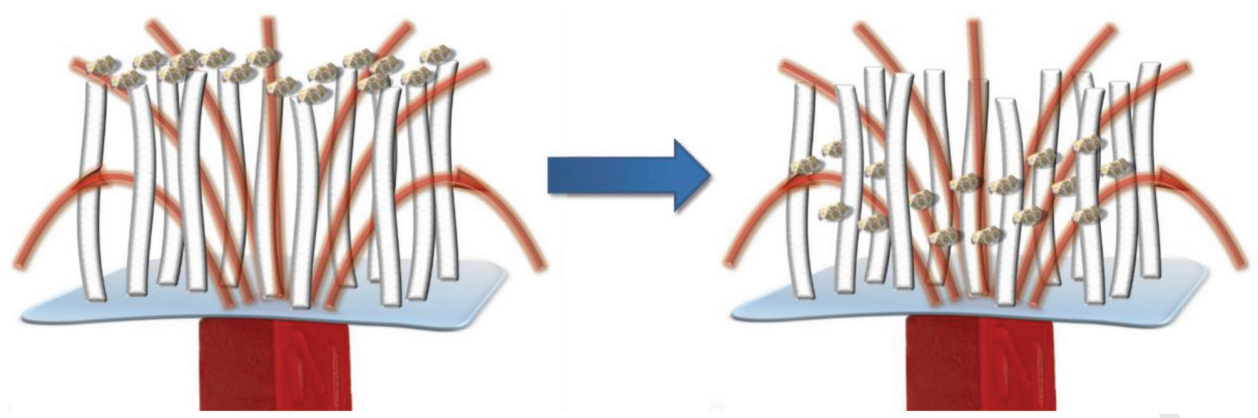

Figure 1. Schematic depicting the setup adapted in this work. The vertically aligned carbon nanotube and magnetic nanoparticles dispersed on top the structure is kept on top of the magnet. After a short duration, the magnetic particles are infiltrated inside the CNT forest.
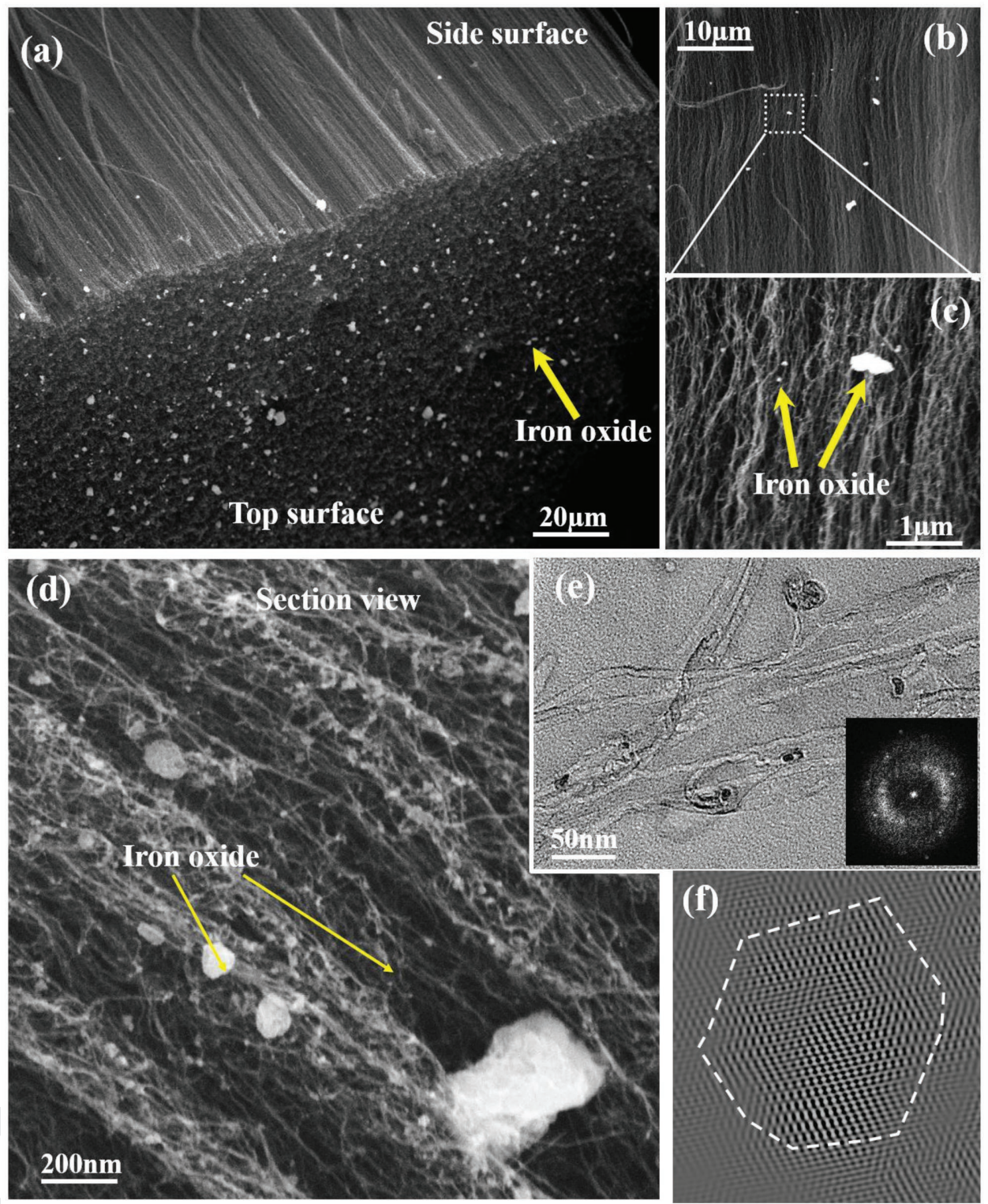

Figure 2. a) Low magnification SEM images of CNT forest in tilted endition to reveal the side and top surface. b) SEM image of the side view and high magnification image selected region c) depicting magnetic particles. d) SEM image after sectioning the forest revealing presence of particles, e) bright field TEM image of particles, and the inset shows FFT of the region. f) Inverse fast Fourief of the particles. 

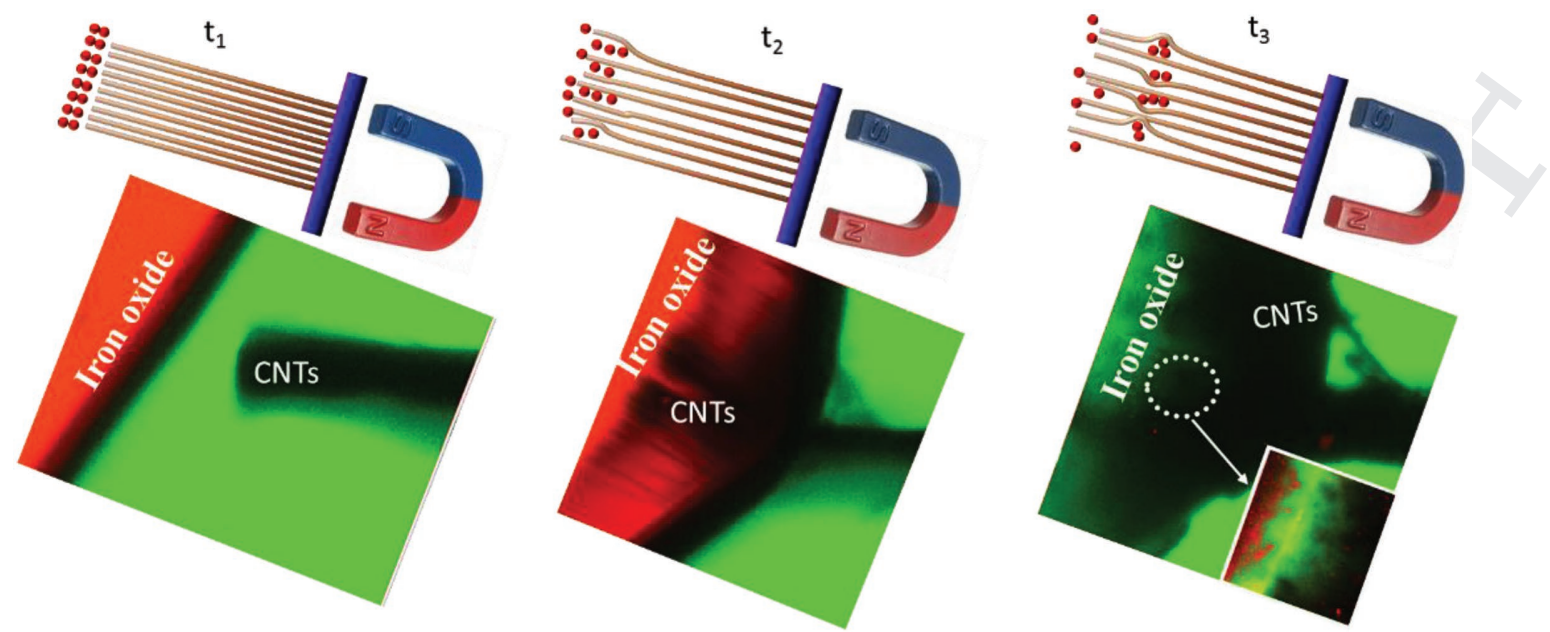

Figure 3. In situ confocal images at different time duration ( $t 1, \mathrm{t} 2$, and $\mathrm{t} 3$ ) of the setup. The schematic on top depicting the observation. The CNT 18 forest in dark and kept on top of the magnet. A high magnification image is shown as an inset.

into the CNTs array with the help of a magnet. We sliced a portion of the CNT forest to observe the cross-sectional view of the array. Figure $2 \mathrm{~d}$ shows the distribution of submicron size particles across the subsurface. A closer observation of the microstructure reveals the possible mechanism of the inclusion of iron oxide into the CNT forest. The particles appear to be entrapped in the intertubular space of the nanotube film (as also displayed in Figure S1, Supporting Information). The applied magnetic field acts on the ferromagnetic particles and attracts those toward the bulk of the nanotube forest. On the other hand, the cohesion force in-between the long and mechanically flexible nanotubes in the forest makes the host structure to become closed again behind the particles thus facilitating trapping of the iron oxide particles. The SEM image of vertically aligned CNT forest grown on CNTs at two different magnifications is shown in Figure S2 of the Supporting Information. We do not observe any particles or contamination on the surface. Figure 2e shows the TEM observation of the MWCNTs with iron oxide particle trapped between them. The Fast Fourier transformed of the MWCNT with particle is shown as inset. The FFT pattern confirms crystallinity of the particle with the $d$-spacing that matches with face-centered cubic $\mathrm{Fe}_{3} \mathrm{O}_{4}$. The inverse Fourier filtered image from these particles shown in Figure $2 \mathrm{f}$ confirms the same.

A systematic time dependent in situ confocal imaging using stained ferromagnetic particles was performed to get more in depth understanding of the infiltration mechanism. Figure 3 shows the schematics as well as the confocal images where the magnetic field was applied for three different time intervals ( $\mathrm{t} 1=0 \mathrm{~min}, \mathrm{t} 2=2 \mathrm{~min}$, and $\mathrm{t} 3=5 \mathrm{~min}$ ). The red color staining has been applied to be able to track the infiltration of the nanoparticles dispersion into the black CNT forest. Initially (at time t1), the CNT forest (black color) was placed near to a droplet of highly concentrated colloidal iron oxide dispersion (red color), while the magnet was kept behind the forest (opposite side to iron oxide). After $2 \mathrm{~min}$, we observed an attraction of the magnetic fluid into the CNT forest due to the magnetic field. In subsequent time interval (t3), we observe the stained red particles from the liquid droplet disappeared as they penetrated into the forest. The inset showing a high magnification image confirms the trapping of particles in the CNT forest. Please note that, in the low 21 magnification image taken at time $\mathbf{3}_{\mathbf{2}}$, the particles are difficult 22 to observe due to their small sizes and black CNT background, 23 although we clearly observe a drastic change in the color of the 24 colloid droplet. The red color particle gets trapped in the black 25 CNT forest and we do not observe red color solution. The time 26 dependent study proves that, as we increase the duration of mag- 27 netic interaction, the amount of infiltrated particles increases. 28 Here we should note that, once ferromagnetic particles infiltrate 29 the forest, they further amplify the local magnetic field and thus 30 accelerate the infiltration process. Furthermore, it is worth clari- 31 fying that, the current experiments are performed in a horizontal 32 arrangement in order to avoid the gravitational effect.

The pristine and $\mathrm{Fe}_{3} \mathrm{O}_{4}$ decorated CNT forest grown on the 34 two different substrates ( $\mathrm{Si}$ and Inconel) were tested for field 35 emission ( $($ vs $E$ ) behavior (Figure 4). We observe a clear reduc- 36 tion of the turn-on fields $\left(E_{\text {to }}\right)$ after incorporating $\mathrm{Fe}_{3} \mathrm{O}_{4}$ parti- 37 cles into the forests. For the samples grown on $\mathrm{Si}$ and Inconel 38 $E_{\text {to }}$ decreased to 0.3 and $0.2 \mathrm{~V} \mathrm{\mu m}^{-1}$, from 2.8 and $3.3 \mathrm{~V} \mathrm{\mu m}^{-1}, 39$ respectively. The Fowler-Nordheim $(\mathrm{F}-\mathrm{N})$ plots displayed in 40 the insets of Figure $4 \mathrm{a}, \mathrm{b}$, indicate linear regimes for the large 41 fields, which is often observed for CNT-based field-emitters. ${ }^{[25]} 42$ Despite the excellent fit, we need to note-since the F-N model 43 has been developed to describe flat metallic surfaces-, one 44 shall use more complex models ${ }^{[27]}$ with additional parameters 45 and statistics that take into account the positions and radii of 46 emitter tips in a network along with their differing work func- 47 tions CNTs $(5.0 \mathrm{eV})$ and $\mathrm{Fe}_{3} \mathrm{O}_{4}(5.3 \mathrm{eV})$ making such a calcula- 48 tion to be beyond the scope of this work.

After the field emission experiment, the nanoparticles 50 were found to be inside the CNT forest, which was verified, 51 by SEM images taken subsequently (Figure S4, Supporting 52 Information). This proves the fact that once the particles are 53 infiltrated into the CNT forest, they are not disturbed by any 54 physical changes (like tilting or inverting the CNT forest). $\quad 55$

As based on current experimental data, it is difficult to probe 56 exact mechanism of the field emission, but based on available 57 literatures, whor for 58 the current field emission behavior. The role of $\mathrm{Fe}_{3} \mathrm{O}_{4}$ particles 59 
(a)

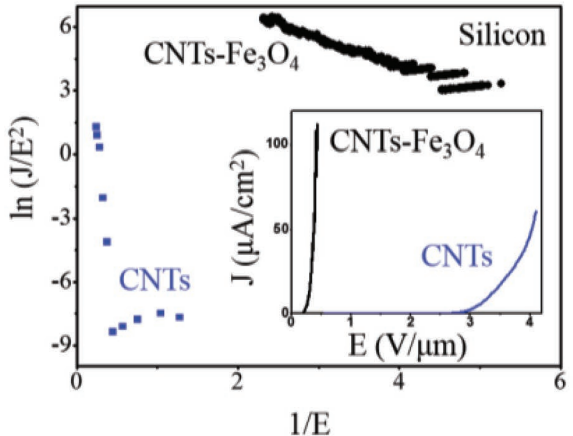

(b)

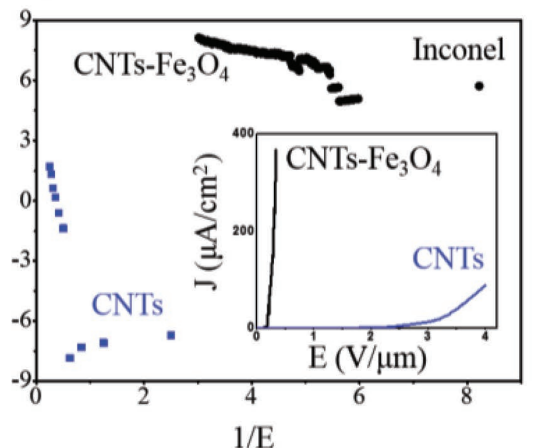

(c)

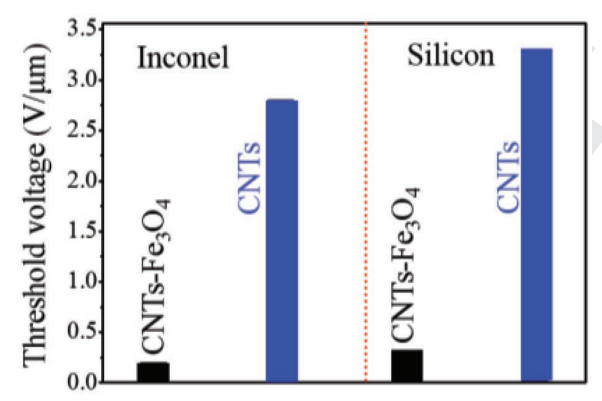

1

2

3

4

5

6

7

8

9

Figure 4. In $\left(J / E^{2}\right)$ versus $1 / E$ for CNT forest grown on a) silicon and b) Inconel with and without magnetic particles. J versus $E$ plot of the samples. The blue color is for CNT and the black color for CNT with particles. c) The threshold voltage for various samples.

is manifold in the field-emission enhancement. First, infiltration of $\mathrm{Fe}_{3} \mathrm{O}_{4}$ nanoparticles results in considerable reduction in the screening effect between the adjacent CNTs. Although, the sharp tips and high aspect ratio are of great advantage for the CNTs to be used as good emitters, the screening effect from the neighboring CNTs compromises these advantages, ${ }^{[28]}$ which is alleviated by the low surface density of $\mathrm{Fe}_{3} \mathrm{O}_{4}$ particles acting as remote emission centers. Second, the nanoparticles form junctions between CNTs making the percolation path more redundant for the electrons across the forest thus improving the overall conductivity of the films, in accordance with electrochemical impedance spectroscopy measurements on different CNT films (Figure S5, Supporting Information). Third, the infiltrated nanoparticles make intimate physical contact with the CNTs, which may be particularly important, when the emitter surface is not entirely conductive (e.g., because of amorphous carbon on the CNTs) thus electrons would need to tunnel through the insulating layer before emitted to the vacuum. And fourth, $\mathrm{Fe}_{3} \mathrm{O}_{4}$ has much higher dielectric permittivity than that of vacuum or air, making the concentration of electric field even more efficient in the location of the nanoparticles similar to that demonstrated earlier for CNTs coated with high permittivity $\mathrm{SrTiO}_{3} \cdot{ }^{[28]}$ However, a significant benefit of $\mathrm{Fe}_{3} \mathrm{O}_{4}$ over $\mathrm{SrTiO}_{3}$ is the high electrical conductivity and good electronic band alignment with MWCNTs, making the injection of electrons into nanoparticles more favored.

\section{Conclusions}

This work demonstrated an easily scalable and simple magnetic method to infiltrate ferromagnetic $\mathrm{Fe}_{3} \mathrm{O}_{4}$ particles into CNT forests without destructing its physical structure. The infiltration is due to attractive forces acting on the ferromagnetic particles in the magnetic field, which is further promoted by the local field of already inserted particles. After infiltration, the excellent localization and entrapment of the nanoparticles are observed, which is due to the mechanical flexibility and to the presence of cohesive forces within the CNT film. The $\mathrm{Fe}_{3} \mathrm{O}_{4}$ decorated CNT forests tested in field-emission experiments display an order of magnitude reduced turn-on field as compared to their pristine counterparts owing to the reduced screening effects, improved conductivity and enhanced electric field localization by the conductive $\mathrm{Fe}_{3} \mathrm{O}_{4}$ emitters having high dielectric permittivity. The current energy efficient and simple method suggests infiltration of any other magnetic particles in virtually any flexible open pore structure host materials for novel sensors, electronics devices, and environmental applications.

\section{Experimental Section}

Vertically aligned MWCNTs were grown on Si and Inconel substrates in a water-assisted CVD system. CNTs grown on Si were around $1 \mathrm{~cm}$ in height and those grown on Inconel were around of $800 \mu \mathrm{m}$ in height. Iron oxide $\left(\mathrm{Fe}_{3} \mathrm{O}_{4}\right)$ particles were synthesized using chemical precipitation method. ${ }^{[26]} 2 \mathrm{mg}$ of these particles was spread across the surface of the CNTs using a spatula. The CNT films with the $\mathrm{Fe}_{3} \mathrm{O}_{4}$ particles on their top were placed in a Petri dish and then a magnet having a magnetic flux density of $\approx 0.1 \mathrm{~T}$ was brought beneath the container and slowly moved across till most of the nanoparticles got infiltrated inside the CNT array.

The micro and crystal structure of the materials are characterized using scanning electron microscopy (FEI Quanta 400 ESEM FEG), HRTEM (JEOL 2100 F TEM), and Raman spectroscopy (Kaiser FiberOptic RAMAN). The in situ confocal microscopy of the process was performed using a Nikon confocal microscope, in order to understand the method of infiltration of nanoparticles into the CNT forest. For this, the iron oxide nanoparticles were mixed with colored dye in water before the infiltration experiments.

After the infiltration of metal nanoparticles; the samples were transferred to a vacuum chamber with a base pressure $<10^{-6}$ Torr to conduct field emission measurements. ITO coated with green phosphorous was used as an anode (sample area of $1 \times 1 \mathrm{~cm}^{2}$ ). The distance between the cathode and the anode was adjusted using a micrometer screw gauge. The CNT tips and the anode were kept $\approx 500 \mu \mathrm{m}$ distance apart. The DC voltage was supplied using a Keithley 2410 device. The EIS measurements were performed using a two electrode setup with the CNT on the substrate as the working electrode and lithium metal as the counter/reference electrode. $1 \mathrm{M} \mathrm{LiPF}_{6}$ in $1: 1 \mathrm{v} / \mathrm{v}$ mixture of ethylene carbonate dimethyl carbonate was used as the electrolyte and glass microfiber filter membrane was used as the separator. The EIS measurements were carried out from $70 \mathrm{kHz}$ to $10 \mathrm{mHz}$ by applying a constant DC bias with a sinusoidal signal of $10 \mathrm{mV}$.

\section{Supporting Information}

Supporting Information is available from the Wiley Online Library or from the author. 


\section{Acknowledgements}

S.S. and C.S.T. contributed equally to this work.

\section{Conflict of Interest}

The authors declare no conflict of interest.

\section{Keywords}

carbon nanotubes, field emission, hybrid architecture, magnetic particle

Received: December 14, 2017

Revised: May 23, 2018 Published online:

[1] M. M. J. Treacy, T. W. Ebbesen, J. M. Gibson, Nature 1996, 381, 678.

[2] E. W. Wong, P. E. Sheehan, C. M. Lieber, Science 1997, 277, 1971.

[3] T. W. Ebbesen, H. J. Lezec, H. Hiura, J. W. Bennett, H. F. Ghaemi, T. Thio, Nature 1996, 382, 54.

[4] H. Dai, J. H. Hafner, A. G. Rinzler, D. T. Colbert, R. E. Smalley, Nature 1996, 384, 147.

[5] H. Tanaka, S. Akita, L. Pan, Y. Nakayama, Jpn. J. Appl. Phys. 2004, 43, 864.

[6] M. S. Fuhrer, J. Nygard, L. Shih, M. Forero, Y. G. Yoon, M. S. Mazzoni, H. J. Choi, J. Ihm, S. G. Louie, A. Zettl, P. L. McEuen, Science 2000, 288, 494.

[7] H. W. C. Postma, T. Teepen, Z. Yao, M. Grifoni, C. Dekker, Science 2001, 293, 76.

[8] P. G. Collins, M. S. Arnold, P. Avouris, Science 2001, 292, 706.

[9] S. E. Lyshevski, Dekker Encyclopedia of Nanoscience and Nanotechnology, CRC Press, New York 2004.
[10] J. Bao, Q. Zhou, J. Hong, Z. Xu, Appl. Phys. Lett. 2002, 81, 4592.

[11] B. K. Pradhan, T. Toba, T. Kyotani, A. Tomita, Chem. Mater. 1998, 10, 2 2510.

[12] K. Kordás, T. Mustonen, G. Toth, J. Vähäkangas, A. Uusimäaki, 4 H. Jantunen, A. Gupta, K. V. Rao, R. Vajtai, P. M. Ajayan, Chem. 5 Mater. 2007, 19, 787

[13] W. Chen, X. Pan, M.-G. Willinger, D. S. Su, X. Bao, J. Am. Chem. 6 Soc. 2006, 128, 3136

[14] J. Li, S. Tang, L. Lu, H. C. Zeng, J. Am. Chem. Soc. 2007, 129, 9401.8

[15] D. R. Kauffman, D. C. Sorescu, D. P. Schofield, B. L. Allen, 9 K. D. Jordan, A. Star, Nano Lett. 2010, 10, 958.

[16] K. Jiang, A. Eitan, L. S. Schadler, P. M. Ajayan, R. W. Siegel, 11 N. Grobert, M. Mayne, M. Reyes-Reyes, H. Terrones, M. Terrones, 12 Nano Lett. 2003, 3, 275.

[17] J. Kong, M. G. Chapline, H. Dai, Adv. Mater. 2001, 13, 1384.

[18] L. Fu, Z. Liu, Y. Liu, B. Han, P. Hu, L. Cao, D. Zhu, Adv. Mater. 2005, 15 $17,217$.

[19] H. S. Shin, Y. S. Jang, Y. Lee, Y. Jung, S. B. Kim, H. C. Choi, 16 Adv. Mater. 2007, 19, 2873.

[20] B. Liu, J. Y. Lee, J. Phys. Chem. B 2005, 109, 23783.

[21] Q. Liu, Z. C. Chen, B. Liu, W. Ren, F. Li, H. Cong, H.M. Cheng, 19 Carbon 2008, 46, 1892.

[22] L. Jiang, L. Gao, Chem. Mater. 2003, 15, 2848.

[23] Z. Sun, Z. Liu, Y. Wang, B. Han, J. Du, J. Zhang, J. Mater. Chem. 22 $2005,15,4497$

[24] J. Wan, W. Cai, J. Feng, X. Meng, E. Liu, J. Mater. Chem. 2007, 17, 23 1188.

[25] S. Sridhar, L. Ge, C. S. Tiwary, A. C. Hart, S. Ozden, K. Kalaga, 25 S. Lei, S. V. Sridhar, R. K. Sinha, H. Harsh, K. Kordas, P. M. Ajayan, 26 R. Vajtai, ACS Appl. Mater. Interfaces 2014, 6, 1986.

[26] T. N. Narayanan, A. P. Reena Mary, P. K. Anas Swalih, D. Sakthi 28 Kumar, D. Makarov, M. Albrecht, J. Puthumana, A. Anas, 29 M. R. Anantharaman, J. Nanosci. Nanotechnol. 2011, 11, 1958.

[27] J. He, P. H. Cutler, N. M. Miskovsky, Appl. Phys. Lett. 1991, 59, 1644. 31

[28] A. Pandey, A. Prasad, J. P. Moscatello, M. Engelhard, C. Wang, 32 Y. K. Yap, ACS Nano 2013, 7, 117. (1)

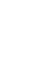

0

